\title{
Bandwidth Constrained Priority-Based Routing Algorithm for Mobile Ad Hoc Networks
}

\author{
Surjeet, Arun Parkash, Rajeev Tripathi \\ Electronics \& Communication Engineering Department, Motilal Nehru National Institute of Technology \\ (MNNIT), Allahabad, India \\ Email: surjeetbalhara@gmail.com, arun@mnnit.ac.in, rt@mnnit.ac.in
}

Received 31 March 2014; revised 30 April 2014; accepted 7 May 2014

Copyright (C) 2014 by authors and Scientific Research Publishing Inc.

This work is licensed under the Creative Commons Attribution International License (CC BY). http://creativecommons.org/licenses/by/4.0/

(c) (i) Open Access

\begin{abstract}
A Mobile Ad hoc NETwork (MANET) is a dynamically formed self-configured network by an autonomous system of mobile nodes connected by wireless links. With the advancements of wireless technology, the necessity of Quality of Service (QoS) is increasing rapidly. Developing QoS constraint routing protocol for MANETs is still a challenging task. As the nodes are free to move randomly, most routing protocols for MANETs are susceptible to node mobility. As routing protocol has to decide which route is able to fulfill the requirement of the desired QoS, routing is the most important part to accomplish the specified application with desired QoS metrics. This paper is based on design of such a kind of proposed MANET algorithm that will estimate the available bandwidth throughout the path by assigning priority. Based on available bandwidth, packets are transferred from source to destination of the applications in queue based on priority. The proposed algorithm is implemented and simulated using NS-2 simulator. Results of our approach show that new protocol can significantly reduce overheads and decrease overall end-to-end delay.
\end{abstract}

\section{Keywords}

AODV, MANETs, NS-2, QoS, Routing

\section{Introduction}

In recent years, there is much advancement in mobile computing and wireless communication technologies that have led to wireless networks offering better connection to mobile users. One important type of such networks is mobile ad hoc network which is infrastructure of less self-configuring network [1]. Each mobile node is capable 
to communicate with other nodes in the network either directly or through multi-hop communication. MANET is used in situations where fixed infrastructure is not available such as natural disaster places, military operations and rescue operation in emergency situations. In an ad hoc network, only those nodes can communicate directly which lie in each other's transmission range.

In other conditions where nodes cannot communicate directly, intermediate nodes act as router for forwarding packets from source to destination. Since the nodes are free to move in a random fashion in MANETs, the network topology may change rapidly and randomly without any prediction. So, a QoS constrained routing protocol should be able to react according to the topology changes. QoS is an assurance to provide some guaranteed constrained parameters services such as delay, jitter, bandwidth and packet delivery ratio etc. There are different protocols that have been proposed for MANETs so far. They are broadly divided into proactive and reactive (on-demand) routing protocols [2]. In case of proactive routing protocols such as DestinationSequenced Distance-Vector routing (DSDV) [3], this updates the network topology information periodically. As nodes move randomly, rapidly and in an arbitrary manner, periodic updates can waste network resources such as bandwidth, battery power consumption etc., reactive routing protocols are more appealing in MANETs as compared to proactive routing protocols. In on-demand routing protocols such as Ad hoc On demand Distance Vector (AODV) protocol [4] and Dynamic Source Routing (DSR) protocol [5], the route is discovered only when source needs to transmit data to the destination. Periodic updates are eliminated in on-demand routing. But these on-demand routing protocols will discover paths with minimum hop count as no QoS provision is there during discovery of path from source to destination.

Generally, while we consider bandwidth constrained path in MANETs, packets will be transferred only if desired bandwidth is available throughout the path. But in case of our proposed Priority Based Routing Algorithm (PBRA), packet transfer proceeds based on priority assigned to multiple applications in queue with different throughput requirements as per bandwidth availability on the path.

The proposed Priority Based Routing Algorithm (PBRA) is based on conventional AODV, in which routing table is used to forward packets and "Hello" messages are used to detect broken route. The protocol modifies and extends AODV [4] to discover the routes and maintain the minimum required bandwidth based on priority of multiple applications in queue. PBRA selects QoS constrained routes in terms of available bandwidth and follows alternate route method for route maintenance. It considers only bandwidth constrained routing based on priority for multiple applications in queue and supports real-time applications. In this paper, a QoS constrained routing has been proposed that provides feedback about the available bandwidth throughout the route with minimized overall overheads during transmission of data.

The rest of this paper is organized as follows: Section 2 brings an overview of the related work. Section 3 presents the details of our proposed QoS routing algorithm and explains route discovery and route maintenance. It also presents the routing algorithm. Section 4 evaluates the performance for Priority Based Routing Algorithm (PBRA) over AODV protocol with different simulation scenarios. Finally, Section 5 concludes the paper with future research directions.

\section{Related Work}

Several routing protocols have been suggested for MANETs [6]-[10] that solve link failure problem, support reliable data transmission, estimating maximum achievable throughput. Considering AODV as base routing protocol, Sung-Ju Lee et al. [6] proposed a backup routing protocol called Ad hoc On demand Distance Vector Backup Routing (AODV-BR). The backup routes are discovered by overhearing Route Reply (RREP) message. If any node is aware of the link failure due to node mobility, packet collision or limited battery during the data transmission, it broadcasts the request control to find a backup node. Zhen Wang et al. [11] proposed a number of issues in QoS routing. Basic problem of QoS routing is finding a feasible path which satisfies QoS parameters as per the requirement of the application. For this, three path computation algorithms are presented for source routing and for hop-by-hop routing. Yan Chen et al. [12] proposed QoS metrics for different network applications which are based on human factors and technology attributes. Both these terms were considered as the key factors that lead the requirements of QoS to vary accordingly. The metrics presented in the paper provided the criteria necessary for QoS assurance. Filali et al. [13] proposed a sniffing based tool technique (called wimeter) that captures and analyzes on real time by which the frames are sent in a preconfigured WLAN. These captured frames are used in determining the portion of time when the channel is free and with this, the available bandwidth is estimated. Call Admission Control Framework is implimented that uses the wimeter as a base of 
bandwidth estimation. Chen and Heinzelman et al. [14] modified the hello messages in the AODV routing protocol so that it carried bandwidth information of each node and its immediate neighbors. This bandwidth information was then used to calculate the residual bandwidth due to second hop neighborhood interference. Two models are proposed for QoS routing. One is adaptive feedback based scheme and other one is admission control scheme. A. Abdrabou et al. [15] proposed a MAC layer based bandwidth estimation method. Bandwidth of a link in discrete time intervals is calculated by averaging the throughputs of the recent packets in the past time window and use this data to estimate the bandwidth in the current time window. This bandwidth estimation method may not be exact as the channel condition may have changed rapidly. S. Soundararajan et al. [16] proposed a new approach which is based on Multipath Routing Backbone (MRB) for supporting enhanced QoS in MANETs. Throughput is significantly improved with minimizing overall end-to-end delay. This protocol is suitable for highly dynamic ad hoc networks where link failures and route breaks occur frequently. It finds multiple disjoint paths from source to destination where each path satisfies the QoS constraints. Wenjing Yan et al. [17] proposed a Greedy based Backup Routing Protocol that considers both route length and link lifetime to achieve high route stability. Primary route for forwarding data packets is formed primarily based on greedy forwarding mechanism, whereas local backup path is established according to link lifetime. Jiazi Li et al. [18] proposed a Multipath Optimized Link State Routing (MP-OLSR) protocol which gives great flexibility by employing different route metrics and cost functions with multipath approach. A modified route recovery and loop detection mechanism is implemented in MP-OLSR to improve QoS. Mammar Sedrati et al. [19] proposed a QoS routing protocol. In this approach, the discovery of the route formation for path reconstruction is done from the source only. A new mechanism to determine multiple disjoint paths for forwarding the packets from source to destination also has been proposed. Nisha Arora et al. [20] proposed Geographic Location Aware Adaptive Routing (GLAAR) which uses node location information as a mean of reducing overall communication overhead for packet forwarding in MANETs. It fetches the node location information using GPS for reducing the computation and communication requirement to select the next node for packet forwarding. Surjeet et al. [21] proposed a modified ad hoc on demand distance vector protocol which gives provision of minimum end to end delay guarantee with assured required throughput. It extends AODV to discover a route with least traffic maintaining the minimum required bandwidth throughout the route. Alternate route method is used for route maintenance.

In summary, many QoS aware routing protocol has been proposed by various authors to improve certain parameters like throughput, delay, jitter and packet delivery ratio. Furthermore at the time of multi applications in queue, these protocols failed to utilize network resources efficiently. Hence, a PBRA based on AODV is proposed in MANETs for QoS provisioning.

\section{QoS Aware Routing}

QoS is a commitment that assures some guaranteed services such as bandwidth, delay, jitter, packet delivery ratio etc. This paper proposes a bandwidth constrained routing on a priority based algorithm for multiple applications in queue with different bandwidth requirement. A QoS based routing has been proposed that provides feedback about the available bandwidth throughout the route, so that data transmission takes place according to available bandwidth which satisfies requirement of the application from multiple applications in queue.

\subsection{Available Bandwidth Estimation}

In bandwidth constrained QoS routing, path is discovered which fulfills the requirement of minimum available bandwidth throughout the route. There are several approaches by which end to end available bandwidth can be calculated. In our approach, end to end available bandwidth is calculated by minimum residual bandwidth among the intermediate nodes throughout the route. As each node shares its available bandwidth between its neighboring nodes, so it is difficult for individual host to calculate residual bandwidth throughout the path. A host will offer bandwidth guaranteed route only if residual bandwidth at a given host is known. However calculation of residual bandwidth through 802.11 MAC is still a difficult problem as the bandwidth is shared among neighbors. Even neighboring hosts are not aware of the traffic status of each other. QoS constrained routing protocol has been proposed by Chen and Heinzelman [14]. Author estimated the residual bandwidth of the host by listening to the channel, the amount of idle time. A new approach has been proposed for calculation of residual bandwidth. 
Let us consider that there are " $n$ " mobile nodes i.e. $N_{1}, N_{2}, N_{3}, \cdots N_{n}$ in a network. Each node has to maintain two hop neighbor routing tables. Firstly, the one hop neighbor table and then the two hop neighbor table. Let us consider a mobile node $N_{x}$ whose one hop neighbor $N_{y}$ and two hop neighbor is $N_{z}$ as shown in Figure 1.

Let consumed bandwidth of $N_{y}$ be $B_{y c o n s}$ and consumed bandwidth $N_{z}$ be $B_{z c o n s}$ for all inflows and outflows in the processes by $N_{y}$ and $N_{z}$. As $N_{y}$ is considered as one hop from $N_{x}$, so there is link directed from $N_{x}$ to $N_{y}$. Similarly there is a link directed from $N_{y}$ to $N_{z}$ as $N_{z}$ is two hop neighbor from $N_{x}$. In order to maintain the two hop neighbor table, these two neighboring host should exchange their one hop table together with consumed bandwidth periodically via control packet $B_{i c o n s}$. $B_{\text {icons }}$ denotes consumed bandwidth by node $N_{i}$ i.e. $i_{\text {th }}$ node. In this, $N_{y}$ and $N_{z}$ have to exchange their routing table together with consumed bandwidth $B_{y c o n s}$ and $B_{z c o n s}$ respectively.

When $N_{y}$ and $N_{z}$ receives $B_{\text {icons }}$ from $N_{x}$ node, these nodes will reply with their consumed bandwidth $B_{y c o n s}$ and $B_{\text {zcons }}$ respectively. Two hop routing table of node $N_{x}$ is shown in Figure 2. When $N_{x}$ has received the currently consumed bandwidth $B_{y c o n s}$ and $B_{z c o n s}$ of both its two hop neighbors $N_{y}$ and $N_{z}$ respectively, the residual bandwidth can be easily estimated by subtracting consumed bandwidth of the two neighboring hops from maximum available bandwidth

$$
B_{\text {residual }}=\left(B_{\max }-\sum_{i=0}^{n} B_{\text {icons }}\right) / W_{f}
$$

$B_{\text {residual }}$ is the available residual bandwidth, $B_{\max }$ is maximum available bandwidth across a path and $B_{\text {icons }}$ is bandwidth consumed by node $N_{i} . W_{f}$ is the weight factor as defined in Equation (1). The division of the residual bandwidth by the weight factor $W_{f}$ is done due to 802.11 MAC. The control messages like RTS, CTS and ACK are induced by MAC. These control messages also consumes bandwidth, that's why back off scheme is not suc-

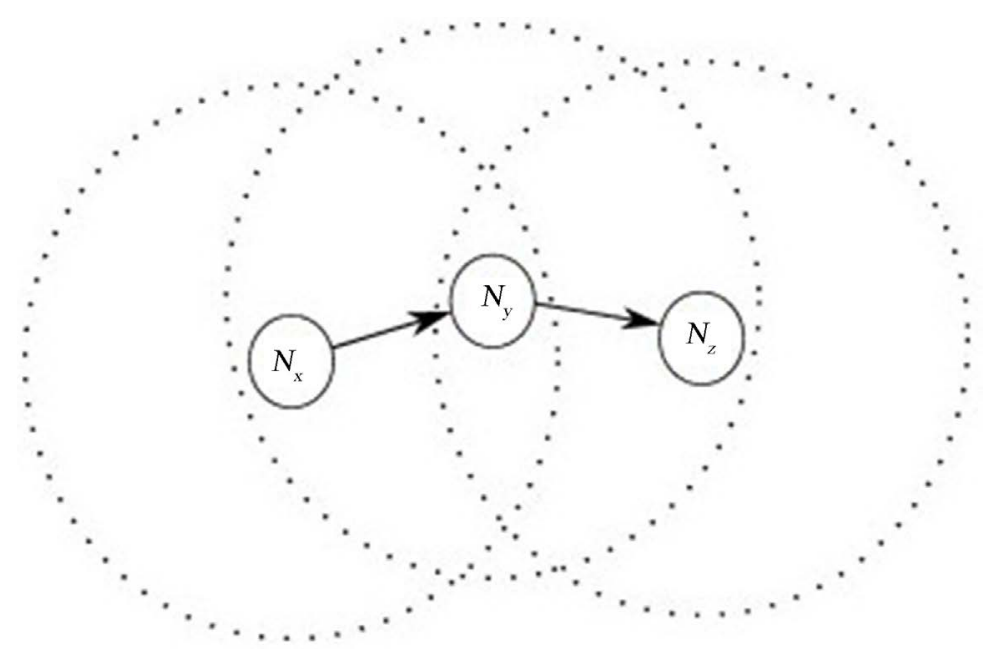

Figure 1. $N_{x}$ and its two hop neighbors $N_{y}, N_{z}[14]$.

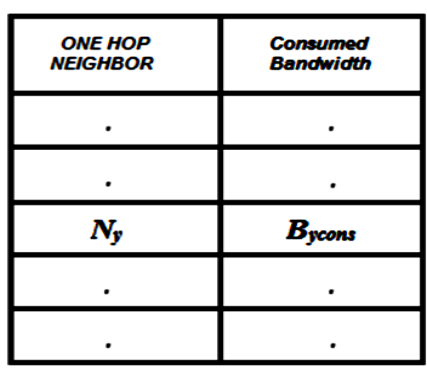

One Hop Neighbor Table of $\boldsymbol{N}_{\boldsymbol{x}}$

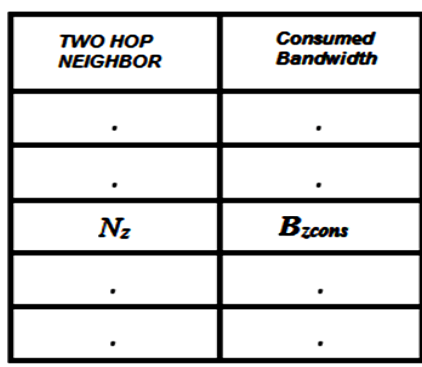

Two Hop Neighbor Table of $\boldsymbol{N}_{\boldsymbol{x}}$

Figure 2. Two hop neighbor table of node $N_{x}$ [14]. 
cessful for use of the entire bandwidth and also, collision of packets can be there.

The weight factor $W_{f}$ can be calculated as

$$
W_{f}=\{\text { RTS }+ \text { CTS }+(\text { Data }+ \text { MAChdr }+ \text { IPhdr })\} / \text { Data }
$$

All the terms like RTS, CTS, $\cdots$ are used to represent the size of these packets respectively. The value of the weight factor calculated above is more as fading errors can cause the retransmission of control or data packets.

\subsection{Proposed Route Discovery}

The proposed priority based QoS aware routing algorithm utilizes cross layer design. For provision of QoS constrained routing in terms of Available Bandwidth (AvBW), extensions are added to Route Request (RREQ), Route Reply (RREP) and RERR messages. Some modifications have been proposed in routing table structure of AODV protocol. Any node which receives the RREQ with QoS guarantee must agree to fulfill the service requirement as desired by the application. To initiate the route discovery process, the source host sends a RREQ packet whose header is changed to include the information about Model, Desired Bandwidt, Least Desired Bandwidth in AODV RREQ header. The whole route discovery procedure is shown in Figure 3. When a host receives a new RREQ, firstly it checks the model. The model indicates whether the required path has to follow Hard QoS or Soft QoS. In case of Hard QoS, packets will be forwarded only when Residual Bandwidth (ReBW) on that link is greater than the Desired Bandwidth (DeBW) on that path and mark this route as Route 1. If available residual bandwidth is less than desired bandwidth, node will discard RREQ. In case of Soft QoS packet will be forwarded when residual bandwidth on that link is greater than the desired bandwidth on that path and mark this route as Route 1 . If residual bandwidth on that path is less than the desired bandwidth but equal to or greater than half of the required bandwidth, desired bandwidth will be updated in RREQ header and will be forwarded. This route is marked as Route 2 and so on until residual bandwidth on the path is less than the Least Required Bandwidth (LrBW).

When the destination host receives the RREQ packet, it will also perform the final checking procedure. Reason for this checking procedure is that if RREP for different routes is sent back through the symmetric links,

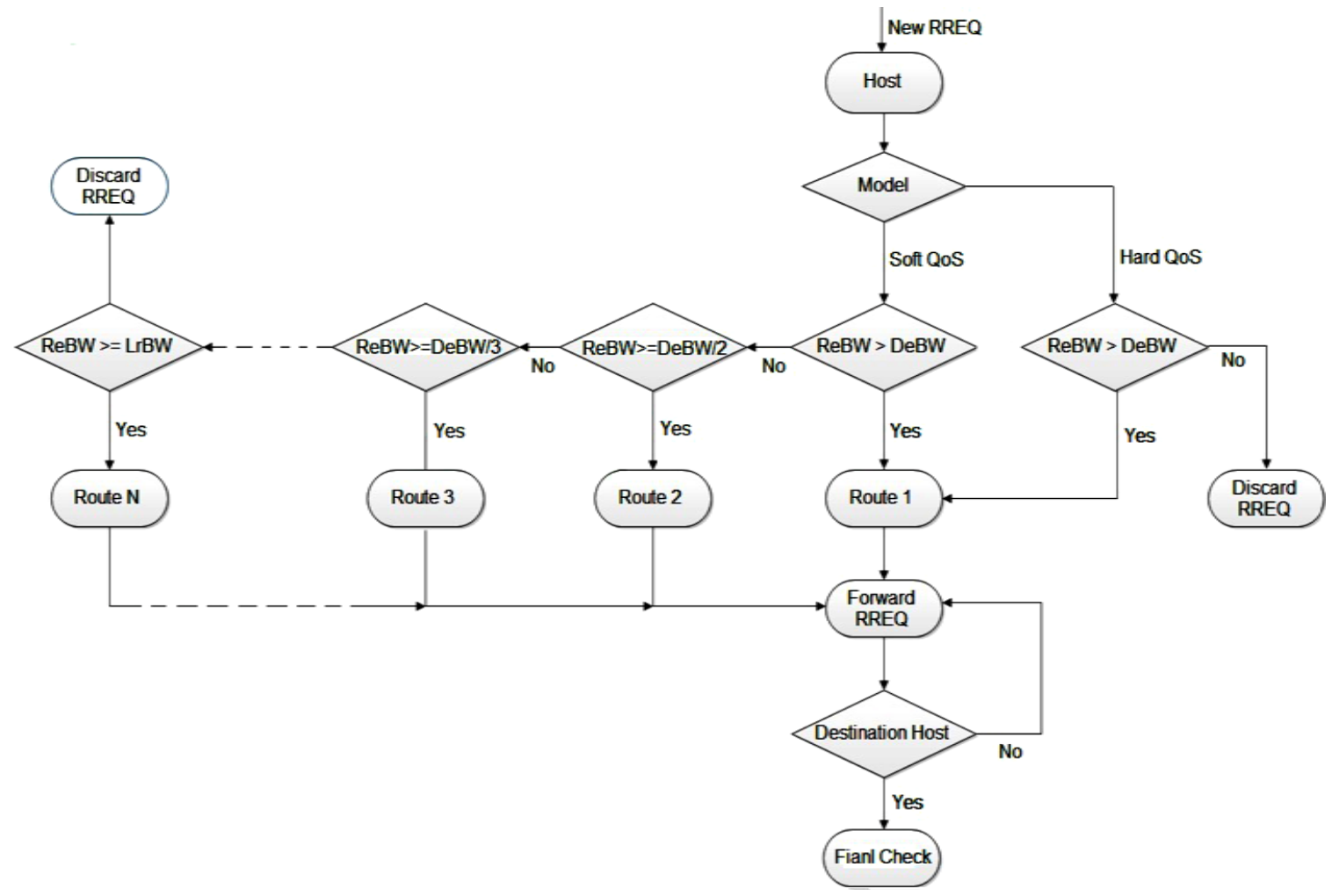

Figure 3. Route discovery procedure. 
the chosen hosts will bring the mutual interference into the network during transmission. Such type of potential interference cannot be taken into consideration during the route discovery procedure. Therefore final check is essential before sending the RREP to the source host.

Finally the destination host sends a RREP with modified header (min bandwidth, AODV RREP header) to the source host through the symmetric links. Once intermediate host receives the RREP, they store it in their route cache and forward the RREPs to the source through the symmetric links.

\subsection{Proposed Route Maintenance}

Broken route in conventional AODV is detected by monitoring the "Hello"' messages. If a node does not receive a "Hello" message from a specific neighbor within a predefined interval, it marks the route as stale thereby invalidating the route and sends a corresponding route error message (RERR) to the upstream hosts. Only the source host reinitiates the route discovery after receiving the error message. Thus using cache memory of the host is not utilized to respond the route break. AODV cannot be implemented in QoS aware routing scheme as bandwidth is not released at the same time whenever there is a route break. It is not possible to calculate the new route without exactly knowing how much bandwidth is consumed by each host in the route. When using our proposed routing scheme, AODV's route maintenance procedure is used with some modifications. If the link between any of the hosts in between the route is broken, it sends an error message to its upstream node. Upstream node will see in its route cache whether there is any alternative path available with same or more residual bandwidth. If yes, it will forward the data through that alternate path. If there is no such path satisfying the QoS constraints with the upstream nodes, source node will get the error message and it will start a new route discovery.

\subsection{Proposed Routing Algorithm}

Determine number of routes from source to destination by assigning priority

Step 1. Assign priority based on bandwidth availability across a link.

Set route request packet $=$ 'flag'

'flag' = True

Step 2. Based on priority discover routes with different bandwidth availability

Step 3. Perform iteration until route break occur \& Route Request packet 'flag' = False

If Route $=1 \quad \& \quad$ RREQ $=+$ ve

Available Bandwidth $=$ Min Bandwidth

else if Route $=2 \quad \& \quad$ RREQ $=+v e$

Available Bandwidth $=$ Minbandwidth 2

else if Route $=3 \quad \& \quad$ RREQ $=+v e$

Available Bandwidth $=$ Minbandwidth 3

else if Route $=4 \quad \& \quad$ RREQ $=+v e$

Available Bandwidth $=$ Minbandwidth/4

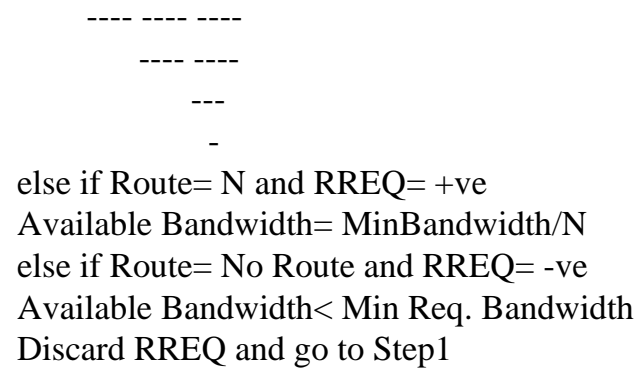

Step 4. $P=\sum_{i=1}^{n} P_{i}$

$P_{i}$ - Path Capacity

$i-i_{\mathrm{th}}$ hop path 
Step 5. If route break occurs then send RERR to upstream nodes and check for alternate path from immediate upstream node's route cache.

Step 6. If no route, then go to Step 1.

\section{Performance Evaluation}

This section investigates the performance of our proposed approach PBRA with conventional AODV. Network simulator NS-2 is used to analyze the performance of proposed PBRA scheme with different weight factors.

\subsection{Performance Metrics}

To compare the performance of our proposed approach, following performance metrics are considered.

Average end-to-end delay refers to the average time taken by the packet to transmit from source to destination.

Average packet delivery ratio is the ratio of number of data packets delivered successfully to the destination compared to the number of data packets actually sent by the source.

Normalized control overhead is the total number of routing packets transmitted per data packet. It can be calculated by total number of routing packets sent to the total number of data packets received.

\subsection{Simulation Environment}

For performance evaluation, nodes are randomly deployed in $1000 \mathrm{~m} \times 1000 \mathrm{~m}$ area. Simulations are carried out using network simulator NS-2. Each node is equipped with a transceiver. Different nodes communicate via radio signals having transmission range of $250 \mathrm{~m}$. Channel bandwidth taken is $2 \mathrm{Mbps}$. Nodes are allowed to move randomly at intervals of $50 \mathrm{~ms}$. In our simulation, IEEE 802.11 is used as MAC layer protocol. The mobility of the nodes is determined by random waypoint mobility model. Path loss model is Two Ray Ground model. For Constant Bit Rate (CBR) data sessions, node pairs are randomly selected with each CBR session generating 5 packets per second with 512 bytes as each data packet size. Table 1 gives the list of simulation parameters used for analysis of our proposed approach.

In order to analyze the performance of our proposed routing protocol with different weight factors and compare with conventional AODV protocol, number of mobile nodes taken in our simulation is 50 nodes.

\subsection{Simulation Results and Discussion}

Initially load in the network is varied from 0.1 Mbps, 0.2 Mbps, 0.3 Mbps, 0.4 Mbps and 0.5 Mbps. Number of

Table 1. Simulation parameters.

\begin{tabular}{cc}
\hline PARAMETER & VALUE \\
Simulation area & $1000 \mathrm{~m} \times 1000 \mathrm{~m}$ \\
Number of nodes & Random waypoint model \\
Mobility model & $20 \mathrm{~m} / \mathrm{sec}$ \\
Mobility & Two ray ground \\
Path loss model & $2 \mathrm{Mbps}$ \\
Channel bandwidth & $250 \mathrm{~m}$ \\
Transmission range & $512 \mathrm{bytes}$ \\
Packet size & IEEE $802.11 \mathrm{DCF}$ \\
MAC protocol & $100 \mathrm{~s}$ \\
Simulation time & 10 \\
CBR data sessions & 5 packets per sec \\
CBR data rate &
\end{tabular}


nodes taken for simulations are 50 nodes. Simulations are carried out by taking different weight factors for PBRA as 1.0, 1.2 and 1.4.

\section{1) Average End-to-End Delay}

From Figure 4, it can be observed that average end-to-end delay in our proposed approach is much less as compared with conventional AODV with different number of nodes and mobility. The end-to-end delay of our proposed node-disjoint scheme also shows improvement in end-to-end delay with increase of weight factor from 1.0 to 1.2 to 1.4 as shown. In this approach, end-to-end delay is significantly minimized as low link failure is there in PBRA as compared to AODV.

\section{2) Normalized Control Overhead}

Figure 5 shows normalized control overhead for PBRA with different weight factors and AODV protocol. Overheads increases with increase in load for both PBRA and AODV due to more frequent route failures. Control overheads are much less in PBRA with different weight factors as compared to AODV as can be seen in Figure 5.

\section{3) Packet Delivery Ratio}

Packet delivery ratio of the proposed protocol compared with AODV protocol is shown in Figure 6. It can be seen from the figure that despite of dynamic nature of MANETs due to mobility, PBRA maintains high degree of packet delivery ratio as compared to AODV protocol due to presence of multiple paths to destination for different bandwidth requirement. When an active route path fails due to mobility of nodes, this approach can maintain the data transfer between source and destination by getting an alternate route if available from one hop upstream node. Thus PBRA approach has much better packet delivery ratio as compared to AODV protocol.

This approach assures high packet delivery ratio even in high mobility.

From the above results, it can be concluded that PBRA shows much significant performance improvement in MANETs. Therefore this approach is a good solution for provisioning of QoS in MANETs for priority based bandwidth estimation for different applications in queue. Due to data transfer according to bandwidth availability, the service quality is much better as compared to AODV. As PBRA discovers bandwidth constrained paths,

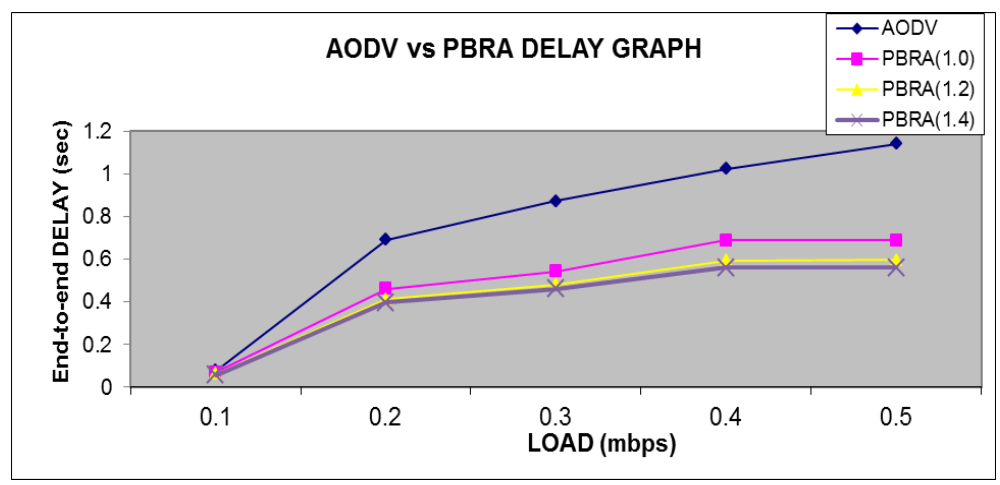

Figure 4. Average end-to-end delay v/s load (50 nodes).

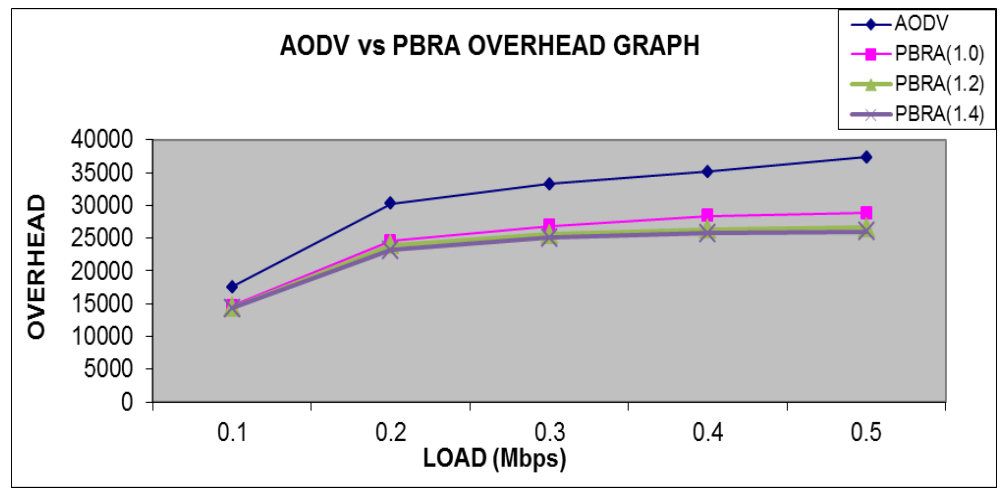

Figure 5. Normalized control overheads v/s load (50 nodes). 


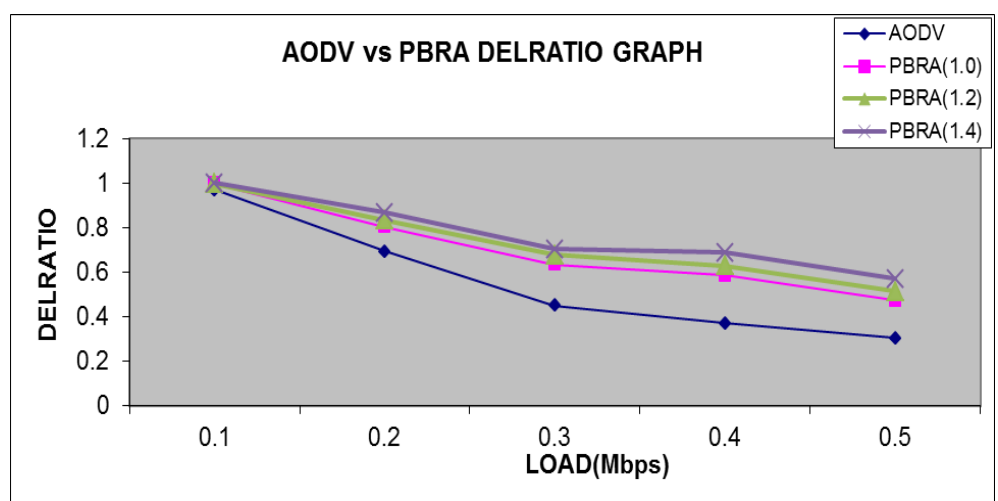

Figure 6. Packet delivery ratio v/s load (50 nodes).

it outperforms AODV for different QoS parameters like end-to-end delay, packet delivery ratio and normalized control overheads.

\section{Conclusions}

In this paper, an on-demand QoS bandwidth constrained routing algorithm for priority-based bandwidth estimation in MANETs has been proposed. The proposed scheme is based on AODV protocol. Conventional AODV is modified to overcome some shortcomings of AODV protocol. This proposed scheme is much effective where networks are not very stable since it can better estimate the residual bandwidth in case of frequent route breaks. Our proposed protocol discovers multiple routes based on bandwidth availability in addition to hop count only. Route maintenance is more efficient than the existing standards of AODV. These characteristics make the protocol more suitable for real-time data and voice transmission applications in MANETs under 802.11. Simulation results have shown significant improvements in terms of certain QoS parameters like end-to-end delay, control overheads and packet delivery ratio for different node mobility.

QoS is the most important issue for latest computer networks. As MANETs follow a distributed and uncertain environment, prioritized QoS is more suitable for such networks. Frequent link failure is a major issue in MANETs, and therefore alternate route strategies should be implemented as per QoS requirements.

In this paper, only bandwidth estimation with priority has been considered for QoS routing. It can be extended to some other resource reservation scheme also. To support QoS with greater reliability and extension, types for use in control messages should also be defined.

\section{References}

[1] Perkins, C.E. (2001) Ad Hoc Network. Addsion-Wesley, Switzerland, 29-51.

[2] Chlamtac, I., Conti, M. and Liu, J.N. (2003) Mobile Ad Hoc Networking: Imperatives and Challenges. Ad Hoc Networks, 1, 13-64. http://dx.doi.org/10.1016/S1570-8705(03)00013-1

[3] Perkins, C.E. and Bhagwat, P. (1994) Highly Dynamic Destination-Sequenced Distance-Vector Routing (DSDV) for Mobile Computers. Proceedings of the ACM SIGCOMM, London, 31 August-2 September 1994, 234-244.

[4] Perkins, C.E. and Royer, E.B. (1999) Ad Hoc on Demand Distance Vector (AODV) Routing. Proceedings of $2^{\text {nd }}$ IEEE Workshop on Mobile Computing System and Application, New Orleans, 25-26 February 1999, 90-100. http://dx.doi.org/10.1109/MCSA.1999.749281

[5] Johnson, D.B., Maltz, D.A. and Broch, J. (2001) DSR: The Dynamic Source Routing Protocol for Multi-Hop Wireless Ad Hoc Networks. Ad Hoc Networking, 5, 132-172.

[6] Lee, S.J. and Gerla, M. (2000) AODV-BR: Backup Routing in Ad Hoc Networks. 2000 IEEE Wireless Communications and Networking Confernce, 3, 1311-1316.

[7] Lai, W.K., Hsiao, S.Y. and Lin, Y.C. (2007) Adaptive Backup Routing for Ad-Hoc Networks. Computer Communications, 30, 453-464. http://dx.doi.org/10.1016/j.comcom.2006.09.011

[8] Yuan, Y.H., Chen, H.M. and Jia, M. (2005) An Optimized Ad-Hoc on Demand Multipath Distance Vector (AOMDV) Routing Protocol. Proceedings of the Asia-Pacific Conference on Communications, Perth, 5 October 2005, 569-573.

[9] Ahmed, I., Tepe, K.E. and Singh, B.K. (2010) Reliable Coverage Area Based Link Expiration Time (LET) Routing 
Metric for Mobile Ad Hoc Networks. Ad Hoc Networks, 28, 466-476.

[10] Wang, Y.H., Chuang, C.C., Hsu, C.P. and Chung, C.M. (2003) Ad Hoc on Demand Routing Protocol Setup with Backup Routes. Proceedings of ITRE International Conference on Information Technology: Research and Education, 11-13 August 2003, 137-141.

[11] Wang, Z. and Crowcroft, J. (1996) Quality-of-Service Routing for Supporting Multimedia Applications. IEEE Journal on Selected Areas in Communications, 14, 1228-1234. http://dx.doi.org/10.1109/49.536364

[12] Chen, Y., Farley, T. and Ye, N. (2004) QoS Requirements of Network Applications on the Internet. ACM Journal in Information-Knowledge-Systems Management, 4, 55-76.

[13] Filali, F. (2006) Wimeter: A Novel Technique for Available Bandwidth Estimation in WLANs and Its Assistance in QoS Provisioning. Eurecom Research Report, 1-16.

[14] Chen, L. and Heinzelman, W. (2005) QoS-Aware Routing Based on Bandwidth Estimation for Mobile Ad Hoc Networks. IEEE Journal of Selected Areas in Communication, 23, 561-572. http://dx.doi.org/10.1109/JSAC.2004.842560

[15] Abdrabou, A. and Zhuang, W. (2006) A Position-Based QoS Routing Scheme for UWB Mobile Ad Hoc Networks. IEEE Journal of Selected Areas in Communication, 24, 850-856. http://dx.doi.org/10.1109/JSAC.2005.863867

[16] Soundararajan, S. and Bhuvaneswaran, R.S. (2011) Multipath Routing Backbone for Improving QoS in Mobile Ad Hoc Networks. European Journal of Scientific Research, 53, 222-230.

[17] Yang, W., Yang, X., Yang, S. and Yang, D. (2011) A Greedy-Based Stable Multi-Path Routing Protocol in Mobile Ad Hoc Networks. Ad Hoc Networks, 9, 662-674. http://dx.doi.org/10.1016/j.adhoc.2010.09.004

[18] Yi, J., Adnane, A., David, S. and Parrein, B. (2011) Multipath Optimized Link State Routing for Mobile Ad Hoc Networks. Ad Hoc Networks, 9, 28-47. http://dx.doi.org/10.1016/j.adhoc.2010.04.007

[19] Sedrati, M., Bilami, A. and Benmohamed, M. (2011) M-AODV: AODV Variant to Improve Quality of Service in MANETs. International Journal of Computer Science Issues, 8, 429-436.

[20] Arora, N. and Jangra, A. (2012) GLAAR: Geographic Location Aware Adaptive Routing in Mobile Ad Hoc Networks. International Journal of Computer Applications, 50, 17-22.

[21] Surjeet, Parkash, A. and Tripathi, R. (2013) QoS Bandwidth Estimation Scheme for Delay Sensitive Applications in MANETs. Journal of Communications and Network, 5, 1-8. http://dx.doi.org/10.4236/cn.2013.51001

\section{Abbreviations}

RTS: Request to Send

CTS: Clear to Send

MAC: Media Access Control

MAChdr: Media Access Control Header

IPhdr: Internet Protocol Header 